\title{
On the Theory of Stable Mode of Dendritic Growth in the Presence of Convective Heat and Mass Transfer Boundary Conditions
}

\author{
DMITRI V. ALEXANDROV \\ Ural Federal University \\ Department of Theoretical and \\ Mathematical Physics \\ Laboratory of Multi-Scale \\ Mathematical Modeling \\ Lenin Avenue 51, 620000 Ekaterinburg \\ RUSSIAN FEDERATION \\ dmitri.alexandrov@urfu.ru
}

\author{
LIUBOV V. TOROPOVA \\ Ural Federal University \\ Department of Theoretical and \\ Mathematical Physics \\ Laboratory of Multi-Scale \\ Mathematical Modeling \\ Lenin Avenue 51, 620000 Ekaterinburg \\ RUSSIAN FEDERATION
}

\author{
PETER K. GALENKO \\ Friedrich-Schiller Universität Jena \\ Physikalisch-Astronomische Fakultät \\ Löbdergraben 32, D-07743 Jena \\ GERMANY
}

\begin{abstract}
The dendritic form is one of the most common forms of crystals growing from supercooled melts and supersaturated solutions. In recent decades, an analytical theory has been developed that describes a stable dendrite growth mode under the conditions of a conductive heat and mass transfer process. However, in experiments, the growth of dendritic crystals is often observed under the conditions of convective fluid flow. In the present work, the theory of the growth of dendritic crystals is developed taking into account the convective mechanism of heat and mass transfer at the crystal-melt interface. A stable mode of dendritic growth in the case of intense convective flows near the steady-state growing dendritic tip is analyzed. The selection theory determining a stable growth mode in the vicinity of parabolic solutions as well as the undercooling balance condition are used to find the dendrite tip velocity and its tip diameter as functions of the melt undercooling. It is shown that the theoretical predictions in the case of convective boundary conditions are in agreement with experimental data for small undercoolings. In addition, the convective and conductive heat and mass transfer mechanisms near the growing dendritic surfaces are compared. Our calculations show that the convective boundary conditions essentially influence the stable mode of dendriticgrowth.
\end{abstract}

Key-Words: Dendritic growth, Forced convection, Selection theory

Received: October 13, 2019. Revised: February 17, 2020. Accepted: March 14, 2020. Published: March 31, 2020.

\section{Introduction}

Dendrites are one of the most common morphological formations that appear as a result of crystallization from the metastable liquid state of melts and solutions [1-8] (figure 1). The shape and kinetics of dendritic crystals depend on the anisotropy of the solid/liquid interface and the processes of heat and mass transfer [9-11]. The experimental data demonstrate that the internal structure of crystallized melts and solutions strongly depends on the metastability degree (undercooling or supersaturation), the crystallization rate, and the characteristic diameter of the dendrite vertices [12-14]. Convection in the liquid phase also plays an important role in the growth of dendritic crystals, changing the conditions of heat and mass trans- fer and leading to mechanical deformations of growth structures [15-17].

The authors of papers [18-20] show that convective fluid flows increase the crystallization rate, compress the thermal and concentration boundary layers, and also lead to an increase in the thermal and concentration gradients in front of growing dendritic crystals. These physical features are usually not taken into account in dendritic growth models. So, for example, a dendritic growth model is usually used with boundary conditions of the conductive type at the crystal/liquid interface [13,21-28]. Such models describe a large number of experimental data for various Péclet and Reynolds numbers under the assumption of laminar flow in the liquid phase (or in the absence of flow). 


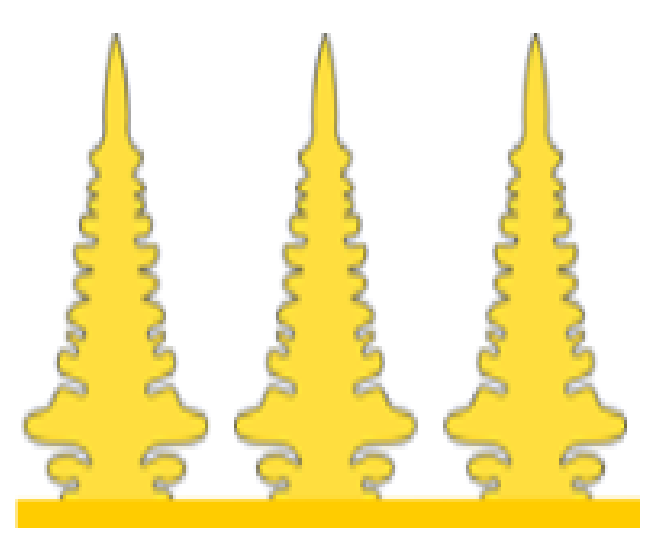

Figure 1: A scheme of dendritic crystals.

However, there are experimental data on dendritic growth in liquids with intense (turbulent) flows at the interfacial boundaries of growing crystals $[29,30]$. In this case, the mechanism of heat and mass transfer ceases to be conductive and is described by the boundary conditions of the convective type $[29,30]$.

This article discusses the influence of the boundary conditions of convective heat and mass transfer at the interfacial boundaries of dendritic crystals on the crystallization process. Namely, in this work, the theory of microscopic solvability is compared with experimental data on the growth of dendritic crystals from $\mathrm{Ti}_{45} \mathrm{Al}_{55}$ melts [19].

\section{Stability Criterion for Convective Heat and Mass Transport}

In this work, we will consider a low-speed crystallization process with intense fluid motion near the dendritic surface. In this case, the process of heat and mass transfer at the interface can be considered as convective, and the boundary conditions of the balance of heat and mass can be written in the form of convective-type conditions [29-31]. To find a selection criterion for a stable dendritic growth mode, we will use the boundary conditions in the presence of convective heat and mass transfer [31]. This type of heat and mass transfer mechanism occurs due to the turbulent flows near the solid/liquid interface $[29,30]$ or as a result of thermo-electrical hydrodynamic fluxes $[12,18]$ shown in figure 2 .

The selection criterion determining a stable combination between the dendrite tip velocity $V$ and its tip diameter $\rho$ is given by [32]

$$
\sigma^{*} \equiv \frac{2 d_{0} D_{T}}{\rho^{2} V}=\frac{2 \sigma_{0} \sqrt{\alpha_{d}} D_{T} \beta_{1}}{\rho}
$$

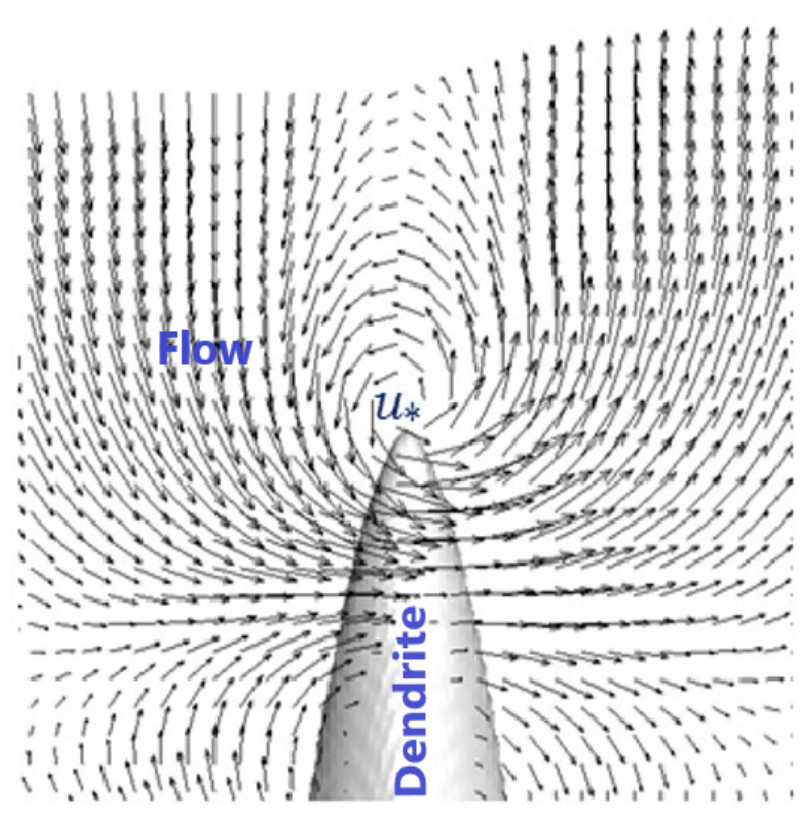

Figure 2: A scheme of dendritic growth in the presence of inrense convective flow. Arrows show the flow velocity.

$$
+\frac{\sigma_{0} \alpha_{d}^{5 / 4}\left(1+b D_{T} \beta_{1}\right)\left(1+\mu \tilde{\tau}_{1}^{3 / 2}\right)^{2}}{\left[1+\nu_{1}\left(\rho b \alpha_{d}^{3 / 4}+\frac{3 \alpha_{d}^{1 / 4} P_{g} \beta_{1} D_{T}}{2^{1 / 4} d_{0}}\right)\right]^{2}}
$$

Here

$$
\begin{gathered}
\tilde{\tau}_{1}=\frac{\alpha_{d}^{1 / 4} \rho b^{2} d_{0}}{2^{1 / 4} P_{g}\left(1+b D_{T} \beta_{1}\right)}, \beta_{1}=\beta_{0}+\frac{m C_{i}\left(1-k_{0}\right)}{T_{Q} \alpha_{s} u_{*}}, \\
b=\frac{\alpha_{h} \rho_{l} c_{l} u_{*}}{2 k_{s}}, \nu_{1}^{2}=\frac{2^{9 / 2} 25 \sigma_{0}}{27}, \\
C_{i}=\frac{\alpha_{s} u_{*} C_{l \infty}}{\alpha_{s} u_{*}-\left(1-k_{0}\right) V}, P_{g}=\frac{\rho V}{2 D_{T}},
\end{gathered}
$$

where $\sigma_{0}$ is the solvability constant, $d_{0}$ is the capillary constant, $\rho_{l}$ is the density of liquid, $k_{0}$ is the solute segregation coefficient, $\alpha_{d}$ is the surface energy stiffness, $\beta_{0}$ is the kinetic growth coefficient, $\mu$ is the selection parameter, $m$ is the liquidus slope, $c_{l}$ is the heat capacity, $u_{*}$ is the friction velocity, $T_{Q}$ is the hypercooling, and $k_{s}$ is the thermal conductivity of solid. Here $\alpha_{h}$ and $\alpha_{s}$ are the convective heat and mass transfer coefficients, $\alpha_{h} / \alpha_{s}=\left(D_{T} / D_{C}\right)^{n}$ with $2 / 3<n<4 / 5\left(D_{T}\right.$ and $D_{C}$ represent the thermal diffusivity and diffusion coefficient). 


\section{Undercooling Balance}

The second condition connecting $V$ and $\rho$ is given by the undercooling balance that represents the sum of different contributions. So, the total undercooling $\Delta T$ takes the form [13]

$$
\Delta T=\Delta T_{T}+\Delta T_{C}+\Delta T_{R}+\Delta T_{K}
$$

The thermal contribution $\Delta T_{T}$ can be written out in the form

$$
\Delta T_{T}=T_{i}-T_{l \infty}=\frac{T_{Q} V k_{s}}{\alpha_{h} \rho_{l} c_{l} u_{*} D_{T}},
$$

where $T_{i}$ and $T_{l \infty}$ represent the temperatures at the interface and far from it.

The contribution $\Delta T_{C}$ arising from the solute concentration field reads

$$
\Delta T_{C}=m\left(C_{i}-C_{l \infty}\right)=\frac{\left(1-k_{0}\right) V m C_{l \infty}}{\alpha_{s} u_{*}-\left(1-k_{0}\right) V},
$$

where $C_{i}$ and $C_{l \infty}$ stand for the solute concentrations at the interface and far from it.

The contributions $\Delta T_{R}$ and $\Delta T_{K}$ appearing due to the interface curvature (the Gibbs-Thomson effect) and the attachement kinetics of atoms at the interface take the form

$$
\Delta T_{R}=\frac{4 d_{0} T_{Q}}{\rho}, \Delta T_{K}=\frac{V}{\mu_{k}},
$$

where $\mu_{k}$ is the kinetic coefficient.

Combining expressions (2)-(5), we obtain the following explicit function

$$
\rho(V)=\frac{4 d_{0} T_{Q}}{\Delta T-\Delta T_{T}(V)-\Delta T_{C}(V)-\frac{V}{\mu_{k}}} .
$$

Now combining the selection criterion (1) and the modified undercooling balance (6), we arrive at the following implicit function determining the dendrite tip velocity $V$

$$
\frac{\rho^{2}(V) V}{2 d_{0} D_{T}} \sigma^{*}(\rho(V), V)=1,
$$

where $\sigma^{*}$ is defined by the right-hand side of expression (1).

Thus, expression (7) determines the dendrite tip velocity $V$ as a function of total undercooling $\Delta T$. The dendrite tip diameter $\rho$ as a function of $\Delta T$ can be found from the modified undercooling balance (6).

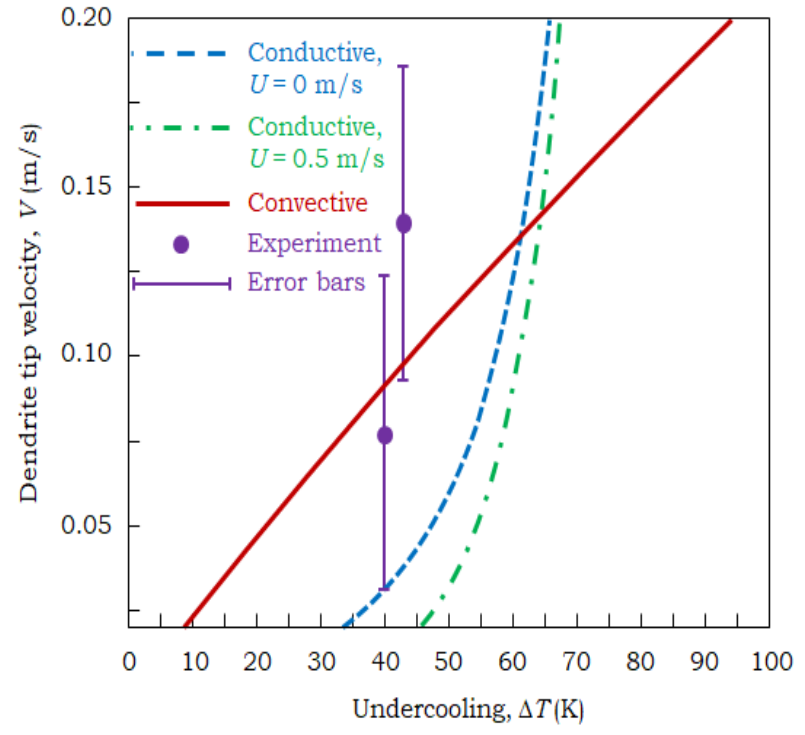

Figure 3: Dendrite tip velocity versus the melt undercooling for $\mathrm{Ti}_{45} \mathrm{Al}_{55}$ alloy. The dashed (blue) and dash-dotted (green) lines show the results for the conductive mechanism of heat and mass transfer in the absence $(U=0 \mathrm{~m} / \mathrm{s})$ and presence $(U=0.5 \mathrm{~m} / \mathrm{s})$ of forced convective flow [34]. The solid line (red) is plotted accordingly to the present model (expression (7)). Theoretical predictions are compared with experimental data [19] for small undercoolings. The model parameters used in calculations are $\sigma_{0}=1.17$, $d_{0}=9.28 \cdot 10^{-10} \mathrm{~m}, D_{T}=2.5 \cdot 10^{-6} \mathrm{~m}^{2} \mathrm{~s}^{-1}$, $\rho_{l}=2.46 \cdot 10^{3} \mathrm{~kg} \mathrm{~m}^{-3}, k_{0}=0.86, C_{l \infty}=55$ at $\%$, $\alpha_{d}=0.3, \beta_{0}=1.88 \cdot 10^{-2} \mathrm{~s} \mathrm{~m}^{-1}, \mu=10^{-3}, m=$ $8.78 \mathrm{~K} \mathrm{at}^{-1}, c_{l}=1237 \mathrm{~J} \mathrm{~kg}^{-1} \mathrm{~K}^{-1}, \alpha_{h}=3.55$, $u_{*}=4 \mathrm{~m} \mathrm{~s}^{-1}, T_{Q}=272.64 \mathrm{~K}, k_{s}=29.22 \mathrm{~W} \mathrm{~m}^{-1}$ $\mathrm{K}^{-1}$.

In the case of conductive heat and mass transfer boundary conditions the selection criterion takes the form $[7,32,33]$

$$
\sigma^{*}=\frac{\sigma_{0} \alpha_{d}^{7 / 4}}{1+\tilde{b}\left(\alpha \alpha_{d}^{-3 / 4}\right)^{11 / 14}}
$$

$$
\begin{gathered}
\times\left\{\frac{1}{\left[1+a_{1} \sqrt{\alpha_{d}} P_{g}\left(1+\frac{\delta_{0} D_{T} \beta_{0}}{d_{0}}\right)\right]^{2}}\right. \\
\left.+\frac{2 m C_{i}\left(1-k_{0}\right) D_{T}}{\left[1+a_{2} \sqrt{\alpha_{d}} P_{C}\left(1+\frac{\delta_{0} D_{C} \beta_{0}}{d_{0 C D}}\right)\right]^{2} T_{Q} D_{C}}\right\},
\end{gathered}
$$


(a)

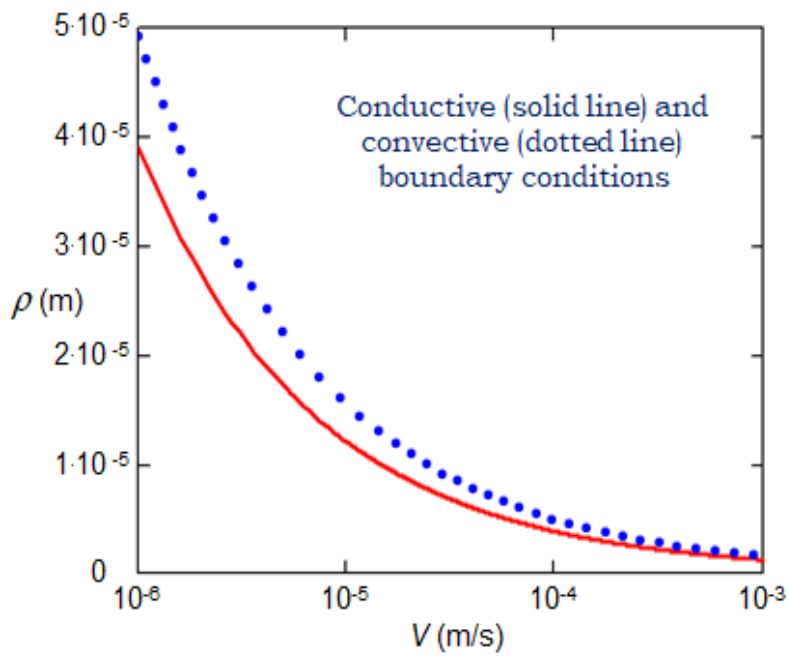

(b)

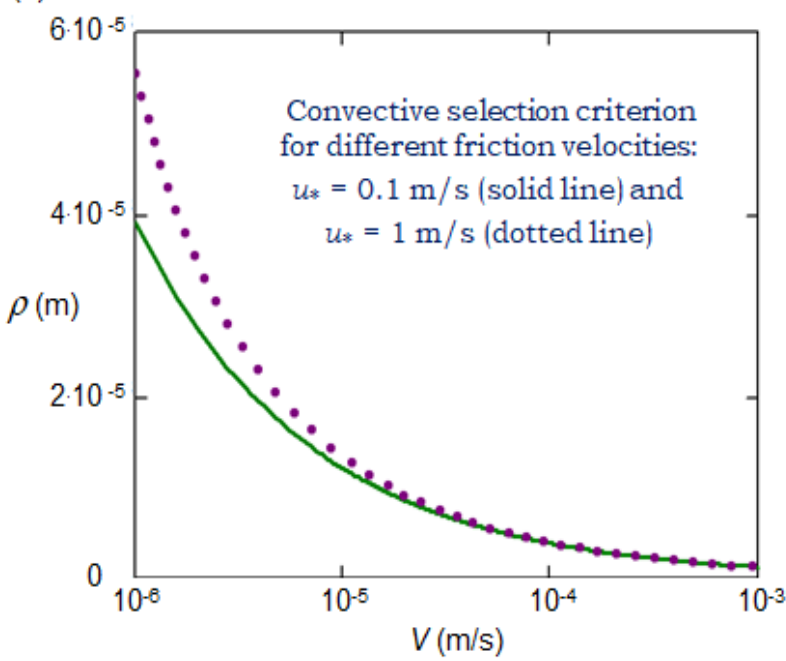

Figure 4: The dendrite tip diameter versus its tip velocity. Panel (a) compares the convective and conductive heat and mass transfer mechanisms. Panel (b) shows the ingluence of friction velocity on $\rho$ and $V$. Parameters used in calculations are $\alpha_{h}=0.0095$, $k_{s}=2.03 \mathrm{~W} \mathrm{~m}^{-1} \mathrm{C}^{-1}, \rho_{l}=10^{3} \mathrm{~kg} \mathrm{~m}^{-3}, c_{l}=4187$ $\mathrm{W} \mathrm{s} \mathrm{kg}{ }^{-1}, d_{0}=2.8 \cdot 10^{-10} \mathrm{~m}, \alpha_{d}=0.35, \sigma_{0}=0.17$, $D_{T}=1.17 \cdot 10^{-7} \mathrm{~m}^{2} \mathrm{~s}^{-1}, \mu=0, \beta_{1}=\beta_{0}=0$. where

$$
\begin{gathered}
a_{1}=\left(\frac{8 \sigma_{0}}{7}\right)^{1 / 2}\left(\frac{3}{56}\right)^{3 / 8}, P_{0}=P_{g}+P_{f}, \\
P_{C}=\frac{\rho V}{2 D_{C}}, a_{2}=\sqrt{2} a_{1}, \\
\alpha=\frac{a d_{0} U}{4 \rho V P}+\frac{a d_{0} U D_{T}}{2 \rho V P D_{C}}, P=1+\frac{2 m C_{i}\left(1-k_{0}\right) D_{T}}{D_{C} T_{Q}}, \\
C_{i}=\frac{C_{l \infty} D_{C}}{D_{C}-\left(1-k_{0}\right) \exp \left(\frac{P_{0} D_{T}}{D_{C}}\right) P_{g} I_{C}(\infty) D_{T}}, \\
a(\Re)=\sqrt{\frac{\Re}{2 \pi}} \frac{\exp (-\Re / 2)}{\operatorname{erfc} \sqrt{\Re / 2}} \text { in } 2 \mathrm{D}, \\
a(\Re)=\frac{\exp (-\Re / 2)}{E_{1}(\Re / 2)} \text { in } 3 \mathrm{D}, \\
\delta_{0}=20 \sqrt{\frac{7}{24}}\left(\frac{56}{3}\right)^{3 / 8}, E_{1}(q)=\int_{q}^{\infty} \frac{\exp (-u)}{u} d u,
\end{gathered}
$$

$U$ is the fluid velocity at infinity, $P_{f}=\rho U /\left(2 D_{T}\right)$, $\tilde{b}$ is the stability constant, $\Re=\rho U / \nu$ is the Reynolds number, $\nu$ is the kinematic viscosity, and $I_{C}(\infty)$ is determined in [32].

Criterion (8) should be supplemented with the corresponding undercooling balance condition, which coincides with expression (2) with contributions $\Delta T_{T}$, $\Delta T_{C}, \Delta T_{R}$, and $\Delta T_{K}$ found in [32] for two- and three-dimensional geometries.

The convective and conductive heat and mass transfer conditions [34] are compared with experimental data [19] in figure 3. Note that the theoretical predictions obtained in [34] are in good agreement with experiments only in the range of moderate and large undercoolings (see, for details, [34]). If we are dealing with the case of small growth velocities, the theory based on conductive-type boundary conditions does not agree with experimental data (the theoretical curves shown in figure 3 by the dashed and dashdotted lines lie outside of the experimental error bars). However, the convective heat and mass transfer mechanism at the solid/liquid boundary leads to the function $V(\Delta T)$ (solid line in figure 2) that corresponds to the experimental error bars at small undercoolings. Indeed, in the case of intense fluid flows around the growing dendrites, one can expect a transition from the laminar regime to the turbulent regime in levitated droplets [35]. This transition can be explained by the fact that the intense fluid curls lead to the convective 
heat and mass transfer mechanism in the vicinity of dendritic tips where the flow is strongly turbulent [35].

In figure 4, we illustrate the influence of convective boundary conditions on dendritic growth. The convective curve (dotted line) lies above the corresponding conductive curve (solid line). In other words, the dendrite tip diameter is greater in the case of convective heat and mass transfer than in the case of the conductive transfer mechanism. The greater values of friction velocity $u_{*}$ also lead to larger values of the dendrite tip diameter.

\section{Conclusion}

In summary, the theory of stable dendritic growth in a binary melt with allowance for the convective heat and mass transfer mechanism at the solid/liquid surfaces of dendritic tips was developed. The theoretical predictions are compared with experiments and previous theory based on the conductive heat and mass transfer boundary conditions. The convective type of heat and mass transfer around the growing solid surfaces arises in a thin boundary layer, where the liquid moves chaotically so that the conductive heat and mass fluxes should be changed by Newtonian boundary conditions. Their main features consist of the fact that the heat and mass fluxes at the growing solid surfaces become convective and depend on the friction velocity $u_{*}$.

To test the theory with convective boundary conditions, we used two equations for the theoretical determination of the dendrite tip velocity and its tip diameter: the selection criterion and the undercooling balance. These equations are reduced to a single one defining an implicit function $V(\Delta T)$. This function was tested against experimental data in the range of small undercoolings. The velocity-undercooling dependence based on the convective heat- and mass transfer mechanism well describes experiments on the solidification of $\mathrm{Ti}_{45} \mathrm{Al}_{55}$ for small undercoolings.

As a final note, the mechanism of heat and mass transfer near the growing dendritic surfaces can be of a mixed type, i.e. it can contain the convective and conductive fluxes. Such a theory containing the mixed type of heat and mass transfer at the dendritic boundary can be developed on the basis of the present analysis and previous theory summarized in [36-39]. This subject represents a new research direction for future investigations.

Acknowledgements: This work was supported by the Russian Science Foundation (grant number 16-1110095).

\section{References:}

[1] R. Trivedi and W. Kurz, Dendritic growth, Int. Mater. Rev. 39, 1994, pp. 49-74.

[2] W. Kurz and D.J. Fisher, Fundamentals of Solidification, Aedermannsdorf, Trans. Tech. Publ. 1989

[3] D. Herlach, P. Galenko and D. Holland-Moritz, Metastable Solids from Undercooled Melts, Amsterdam, Elsevier 2007

[4] P. Pelcé, Dynamics of Curved Fronts, Academic Press, Boston 1987

[5] P.K. Galenko and D.V. Alexandrov, From atomistic interfaces to dendritic patterns, Phil. Trans. R. Soc. A 376, 2018, pp. 20170210.

[6] K. Libbrecht, Snowflakes, Voyageur Press, Minneapolis, MN 2004

[7] D.V. Alexandrov and P.K. Galenko, Thermosolutal and kinetic regimes of an anisotropic dendrite growing under foced convective flow, Phys. Chem. Chem. Phys. 17, 2015, pp. 1914919161.

[8] D.A. Kessler, J. Koplik and H. Levine, Pattern selection in fingered growth phenomena, $A d v$. Phys. 37, 1988, pp. 255-339.

[9] S. Binder, P.K. Galenko and D.M. Herlach, Faceting of a rough solid-liquid interface of a metal induced by forced convection, Phil. Mag. Lett. 93, 2013, pp. 608-617.

[10] P.K. Galenko and D.V. Alexandrov, Selected mode of dendritic growth with n-fold symmetry in the presence of a forced flow, EPL 119, 2017, pp. 16001.

[11] S. Binder, P.K. Galenko and D.M. Herlach, The effect of fluid flow on the solidification of $\mathrm{Ni}_{2} \mathrm{~B}$ from the undercooled melt, J. Appl. Phys. 115, 2014, pp. 053511.

[12] J. Gao, M. Han, A. Kao, K. Pericleous, D.V. Alexandrov and P.K. Galenko, Dendritic growth velocities in an undercooled melt of pure nickel under static magnetic fields: A test of theory with convection, Acta Mater. 103, 2016, pp. 184-191.

[13] P.K. Galenko and D.V. Alexandrov, Dendrite growth under forced convection: analysis methods and experimental tests, Phys.-Usp. 57, 2014, pp. 771-786.

[14] I. Yoshizaki, T. Ishikawa, S. Adachi, E. Yokoyama and Y. Furukawa, Precise measurements of dendrite growth of ice crystals in microgravity, Microgravity Sci. Technol. 24, 2012, pp. 245-253. 
[15] Ph. Bouissou and P. Pelcé, Effect of a forced How on dendritic growth, Phys. Rev. A 40, 1989, pp. 6673-6680.

[16] D.L. Aseev and D.V. Alexandrov, Unidirectional solidification with a mushy layer. The influence of weak convection, Acta Mater. 54, 2006, pp. 2401-2406.

[17] D.A. Saville and P.J. Beaghton, Growth of needle-shaped crystals in the presence of convection, Phys. Rev. A 37, 1988, pp. 3423-3430.

[18] J. Gao, A. Kao, V. Bojarevics, K. Pericleous, P.K. Galenko and D.V. Alexandrov, Modeling of convection, temperature distribution and dendritic growth in glass-fluxed nickel melts, $J$. Cryst. Growth 471, 2017, pp. 66-72.

[19] H. Hartmann, P.K. Galenko, D. Holland-Moritz, M. Kolbe, D.M. Herlach and O. Shuleshova, Non-equilibrium solidification in undercooled $\mathrm{Ti}_{4} 5 \mathrm{Al}_{5} 5$ melts, J. Appl. Phys. 103, 2008, pp. 073509.

[20] P.K. Galenko, K. Reuther, O.V. Kazak, D.V. Alexandrov and M. Rettenmayr, Effect of convective transport on dendritic crystal growth from pure and alloy melts, J. Appl. Phys. 111, 2017, pp. 031602.

[21] P. Pelce and D. Bensimon, Theory of dendrite dynamics, Nucl. Phys. B 2, 1987, pp. 259-270.

[22] E.A. Brener and V.I. Mel'nikov, Pattern selection in two-dimensional dendritic growth, $A d v$. Phys. 40, 1991, pp. 53-97.

[23] E.A. Brener, Pattern formation in threedimensional dendritic growth, Phys. A 263, 1999, pp. 338-344.

[24] E.A. Brener and V.I. Mel'nikov, Twodimensional dendritic growth at arbitrary Peclet number, J. Phys. France 51, 1990, pp. 157-166.

[25] E. Brener, Needle-crystal solution in threedimensional dendritic growth, Phys. Rev. Lett. 71, 1993, pp. 3653-3656.

[26] M. Plapp and A. Karma, Multiscale randomwalk algorithm for simulating interfacial pattern formation, Phys. Rev. Lett. 84, 2000, pp. 17401743.

[27] D.V. Alexandrov and P.K. Galenko, Dendritic growth with the six-fold symmetry: theoretical predictions and experimental verification, $J$. Phys. Chem. Solids 108, 2017, pp. 98-103.

[28] D.V. Alexandrov and P.K. Galenko, Thermosolutal growth of an anisotropic dendrite with six-fold symmetry, J. Phys.: Condens. Matter 30, 2018, pp. 105702.
[29] D.L. Feltham, M.G. Worster and J.S. Wettlaufer, The influence of ocean flow on newly forming sea ice, J. Geophys. Res. 107, 2001, pp. 3009.

[30] D. Notz, M.G. McPhee, M.G. Worster, G.A. Maykut, K. H. Schlünzen and H. Eicken, Impact of underwater-ice evolution on Arctic summer sea ice, J. Geophys. Res. 108, 2003, pp. 3223.

[31] D.V. Alexandrov and I.G. Nizovtseva, To the theory of underwater ice evolution, or nonlinear dynamics of "false bottoms", Int. J. Heat Mass Trans. 51, 2008, pp. 5204-5208.

[32] D.V. Alexandrov, P.K. Galenko and L.V. Toropova, Thermo-solutal and kinetic modes of stable dendritic growth with different symmetries of crystalline anisotropy in the presence of convection, Phil. Trans. R. Soc. A 376, 2018, pp. 20170215.

[33] D.V. Alexandrov and P.K. Galenko, Selection criterion of stable dendritic growth at arbitrary Péclet numbers with convection, Phys. Rev. E 87, 2013, pp. 062403.

[34] P.K. Galenko, D.A. Danilov, K. Reuther, D.V. Alexandrov, M. Rettenmayr and D.M. Herlach, Effect of convective flow on stable dendritic growth in rapid solidification of a binary alloy, J. Cryst. Growth 457, 2017, pp. 349-355.

[35] R.W. Hyers, G. Trapaga and B. Abedian, Laminar-turbulent transition in an electromagnetically levitated droplet, Metall. Mater. Trans. B 34B, 2003, pp. 29-36.

[36] P.K. Galenko, D.V. Alexandrov and E.A. Titova, The boundary integral theory for slow and rapid curved solid/liquid interfaces propagating into binary systems, Phil. Trans. R. Soc. A 376, 2018, pp. 20170218.

[37] D.V. Alexandrov and P.K. Galenko, Selection criterion of stable mode of dendritic growth with n-fold symmetry at arbitrary Péclet numbers with a forced convection, In: IUTAM Symposium on Recent Advances in Moving Boundary Problems in Mechanics, IUTAM Bookseries 34 (eds. S. Gutschmidt, J.N. Hewett and M. Sellier) 34, 2019, pp. 203-215.

[38] D.V. Alexandrov, D.A. Danilov and P.K. Galenko, Selection criterion of a stable dendrite growth in rapid solidification, Int. J. Heat Mass Trans. 101, 2016, pp. 789-799.

[39] D.V. Alexandrov and P.K. Galenko, Selected mode for rapidly growing needle-like dendrite controlled by heat and mass transport, Acta Mater. 137, 2017, pp. 64-70. 